\title{
Fixation time in evolutionary graphs: A mean-field approach
}

\author{
Mahdi Hajihashemi ${ }^{1, *}$ and Keivan Aghababaei Samani ${ }^{1,2, \dagger}$ \\ ${ }^{1}$ Department of Physics, Isfahan University of Technology, Isfahan 84156-83111, Iran \\ ${ }^{2}$ International Institute for Applied System Analysis (IIASA), Schlossolatz 1, A-2361 Laxenburg, Austria
}

(Received 13 August 2018; revised manuscript received 8 March 2019; published 12 April 2019)

\begin{abstract}
Using an analytical method we calculate average conditional fixation time of mutants in a general graphstructured population of two types of species. The method is based on Markov chains and uses a mean-field approximation to calculate the corresponding transition matrix. Analytical results are compared with the results of simulation of the Moran process on a number of network structures.
\end{abstract}

DOI: 10.1103/PhysRevE.99.042304

\section{INTRODUCTION}

Evolutionary graph theory (EGT) [1] is one of the most celebrated methods to study the evolution of species in graph-structured populations. In this theory, one considers a constant-size population of individuals which are connected to each other through a (directed) network which is called evolutionary graph [2]. A fitness is assigned to each type of species. The evolutionary dynamics of this population is determined by a birth-death process in which at each time step, one individual is selected for reproduction with a probability proportional to its fitness. Then, one of its neighbors is selected with a probability determined by evolutionary graph and is replaced by the newborn offspring. This is in fact a generalization of the so-called Moran process [3] which takes place in a graph-structured population instead of a well-mixed population.

This theory has been vastly studied in recent years and its various features and generalizations are addressed. Here we confine ourselves to populations constructed from two types of species whom we call residents and mutants. An interesting process is to start with just one mutant and see what the fate of the system is. In fact, the system will end up in one of the two possible states, namely, fixation or extinction of mutants. Two main quantities corresponding to this process are fixation probability and fixation time. Fixation probability is the probability for a single mutant to take over the whole population and fixation time is the average (conditional) time needed for this result. Both of these quantities are investigated by many researchers [4-10].

One of the key features in such investigations is the interplay between structure and dynamics. Fixation or extinction processes are considerably affected by the structural properties of the underlying network such as dimension and heterogeneity [11-16].

Evolutionary processes on networks are also investigated by other methods such as adaptive dynamics [17] and game theoretical methods [18-21].
Obtaining fixation time is more challenging than fixation probability. In this paper we propose a mean-field approximation to obtain fixation time for various network structures. Using approximations and mean-field methods is prevalent for studying various types of dynamics on networks. For example, in Ref. [22] WKB approximation is used for determining the optimal path to the specific deviation in structured populations. Furthermore, the mean time to fixation and extinction and their corresponding probabilities are evaluated. In another investigation, a mean-field approach is used for approximating average fixation time on a complex network in evolutionary games dynamics [23]. As a final example, in Ref. [24] fluctuating mutants population on a graph is considered as a random walk and then, by using martingales and exploiting symmetry in the steps of the random walk, analytical expressions for fixation probabilities are obtained.

In this paper, we review an analytical method to find conditional fixation and extinction times and exploit it to obtain a mean-field approximation for fixation time on some structured populations, namely cycle and complete graphs, random Erdös-Rényi, scale-free, small-world, and regular networks.

We should emphasize that the birth-death process considered in this paper is just one of the possible update rules for evolutionary dynamics in graph-structured populations. Other update rules such as the death-birth process $[25,26]$ or any diffusion process taking place in a network are exploited as well [27-30]. In Ref. [31] one can find a wide list of dynamical models used in well-mixed and graph-structured populations.

The organization of the paper is as follows. In Sec. II we develop a general method for calculating fixation time based on Markov chains. In Sec. III we apply this method to obtain fixation time for some specific network topologies. Section IV is devoted to summary and concluding remarks.

\section{GENERAL METHOD}

Consider a graph with $N$ nodes. Each node can be of one of two types, namely resident and mutant. Each type

\footnotetext{
*mehdi.hajihashemi@ph.iut.ac.ir
}

†samani@cc.iut.ac.ir 


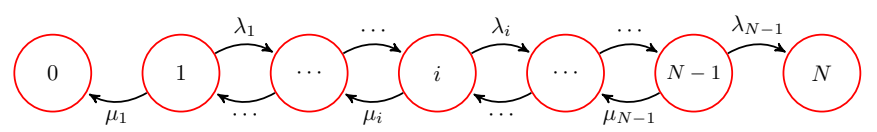

FIG. 1. Markov chain corresponding to the Moran process. The state $i$ represents all configurations with $i$ mutants in the network. The chain transits from state $i$ to state $i+1$ with probability $\lambda_{i}$ and to state $i-1$ with probability $\mu_{i}$. States 0 and $N$ are absorbing states.

has its own fitness which is 1 for residents and $r$ for mutants. A Moran process is running on top of this graph. At each time step, one node is selected for reproduction with a probability proportional to its fitness. Then, one of its neighbors is selected randomly and is replaced by the reproduced offspring. There are two important quantities corresponding to this process. The first one is fixation probability, i.e., the probability for a single mutant to take over the whole network. The second one is the average fixation time, i.e., the average time a single mutant needs to take over the whole network.

Here we introduce a mean-field approach to calculate these two quantities for a category of complex networks using Markov chains.

There is a Markov chain corresponding to the above process. This Markov chain is shown in Fig. 1. Each state of this chain is specified by the number of mutants. The state $i$ represents all configurations of evolutionary graph with $i$ mutants. The transition matrix of this Markov chain is defined as follows:

$$
P_{i j}=\lambda_{i} \delta_{i+1, j}+\mu_{i} \delta_{i-1, j}+q_{i} \delta_{i, j}
$$

Clearly, $\quad \lambda_{i}+\mu_{i}+q_{i}=1 \quad$ and $\quad \lambda_{0}=\mu_{0}=\lambda_{N}=\mu_{N}=0$. States $S_{0}$ and $S_{N}$ are absorbing states. The first one corresponds to extinction of mutants and the second one corresponds to their fixation. In fact, $\lambda_{i}$ is the (average) probability of increasing the number of mutants from $i$ to $i+1$ and $\mu_{i}$ is the (average) probability of decreasing this number from $i$ to $i-1$. To calculate $\lambda_{i}$ and $\mu_{i}$ exactly, one has to take into account all graph configurations with $i, i+1$, and $i-1$ mutants. Generally, this is not an easy task, but as we will show one can obtain them approximately for a number of graph topologies.

The above Markov chain is an example of absorbing Markov chains. There is a well-known method to calculate absorption probabilities and absorption time for this kind of Markov chains [32]. It should be emphasized that absorption time differs from fixation or extinction time. In fact absorption time is a weighted average of fixation and extinction times. We will come back to this point later.

Consider a general absorbing Markov chain. The transition matrix of this chain can be written in the following general form which is called canonical form:

$$
P=\left(\begin{array}{l|l}
Q & R \\
\hline 0 & I
\end{array}\right)
$$

For the transition matrix of Eq. (1) we have

$$
\begin{aligned}
Q & =\left[\begin{array}{ccccc}
q_{1} & \lambda_{1} & & & \\
\mu_{2} & q_{2} & \lambda_{2} & & \\
& \ddots & \ddots & \ddots & \\
& & \mu_{N-2} & q_{N-2} & \lambda_{N-2} \\
& & \\
\mu_{N-1} & q_{N-1}
\end{array}\right], \\
R & =\left[\begin{array}{cc}
\mu_{1} & 0 \\
0 & 0 \\
\vdots & \vdots \\
0 & 0 \\
0 & \lambda_{N-1}
\end{array}\right], I=\left[\begin{array}{ll}
1 & 0 \\
0 & 1
\end{array}\right] .
\end{aligned}
$$

The so-called fundamental matrix corresponding to this transition matrix which is defined as $N=(I-Q)^{-1}$ can be used to calculate absorption probabilities and absorption times. Let us define $t_{i}$ to be the (average) absorption time of the Markov chain starting from state $i$, and $\rho_{i}^{e}$ and $\rho_{i}^{f}$ to be extinction and fixation probabilities starting from state $i$, respectively. We emphasize again that by fixation and extinction we mean absorption to the states $S_{0}$ and $S_{N}$ respectively. Following Ref. [32] we use matrix notation to denote these quantities:

$$
t=\left[\begin{array}{c}
t_{1} \\
t_{2} \\
\vdots \\
t_{N-1}
\end{array}\right], B=\left[\begin{array}{cc}
\rho_{1}^{e} & \rho_{1}^{f} \\
\rho_{2}^{e} & \rho_{2}^{f} \\
\vdots & \vdots \\
\rho_{N-1}^{e} & \rho_{N-1}^{f}
\end{array}\right] .
$$

Then, one can easily obtain absorption probabilities and times using the fundamental matrix as $B=N R, t=N c$, where $c=$ $(1,1, \ldots, 1)^{t}$.

The matrix $(I-Q)$ corresponding to the transition matrix, Eq. (1), is a tridiagonal matrix. Obtaining the inverse of the tridiagonal matrix is straightforward [33]. Particularly, for graphs whose corresponding transition matrices satisfy $\lambda_{i}=$ $r \mu_{i}$, elements of the fundamental matrix read

$$
N_{i j}=\left\{\begin{array}{ll}
\frac{\left(r^{j-1}-1\right)\left(r^{N-i-1}-1\right)}{\mu_{i}(r-1)\left(r^{N}-1\right)} & i \geqslant j \\
\frac{r^{j-i}\left(r^{i-1}-1\right)\left(r^{N-j-1}-1\right)}{\mu_{i}(r-1)\left(r^{N}-1\right)} & i<j
\end{array} .\right.
$$

Fixation and extinction probabilities on graphs with $\lambda_{i}=$ $r \mu_{i}$ can be obtained by direct calculation:

$$
\rho_{i}^{f}=1-\rho_{i}^{e}=\frac{1-\frac{1}{r^{i}}}{1-\frac{1}{r^{N}}} .
$$

Calculating absorption times is also straightforward, but here we are interested not only in absorption times but also in fixation times and extinction times separately. To this end, we need to modify the above method as below.

In the transition matrix of the present problem, $R$ is a $(N-1) \times 2$ matrix and so is the matrix $B$. Now, using each column of matrix $B$ we define a diagonal matrix and call them extinction and fixation matrices respectively:

$$
\begin{aligned}
& E=\operatorname{diag}\left(\rho_{1}^{e}, \ldots, \rho_{N-1}^{e}\right), \\
& F=\operatorname{diag}\left(\rho_{1}^{f}, \ldots, \rho_{N-1}^{f}\right) .
\end{aligned}
$$


Using the above definitions we define

$$
\begin{array}{ll}
Q^{e}:=E^{-1} Q E, & N^{e}:=\left(I-Q^{e}\right)^{-1}, \\
Q^{f}:=F^{-1} Q F, & N^{f}:=\left(I-Q^{f}\right)^{-1} .
\end{array}
$$

We also denote average extinction and fixation times starting with $i$ mutants by $t_{i}^{e}$ and $t_{i}^{f}$, respectively. Then, one can show that these times are obtained as $t^{e}=N^{e} c$ and $t^{f}=N^{f} c$, respectively in a matrix form. Matrix elements of $N^{e}$ and $N^{f}$ are obtained easily using Eq. (6) as

$$
N_{i j}^{e}=N_{i j} \frac{\rho_{j}^{e}}{\rho_{i}^{e}}, N_{i j}^{f}=N_{i j} \frac{\rho_{j}^{f}}{\rho_{i}^{f}},
$$

which is in agreement with the results of Ref. [8]. The general method can be used for Markov chains with more than two absorbing states as well. This may happen in populations with more than two types of species.

Substituting $N_{i j}$ from Eq. (3) and $\rho_{i}^{f}$ from Eq. (4) in the second term of Eq. (7) the fixation time $t_{1}$ can be obtained in a closed form,

$$
t_{1}=\sum_{j=1}^{N-1} \frac{\left(r^{j}-1\right)\left(r^{N}-r^{j}\right)}{(r-1)\left(r^{N}-1\right) \mu_{j} r^{j}},
$$

which is in agreement with the results obtained by other methods $[4,9,34]$.

\section{EFFECT OF NETWORK TOPOLOGY ON FIXATION TIME}

In this section, we apply the method of the previous section to some specific network structures, namely cycle graph, complete graphs, random network (Erdös-Rényi model), scalefree network (Barabasi-Albert model), regular network, and small-world network (Watts-Strogatz model).

We show that in all of these structures the condition $\lambda_{i}=$ $r \mu_{i}$ is fulfilled either exactly or approximately. Therefore, to obtain fixation time one just needs to obtain $\mu_{i}$ and insert it in Eq. (8). In cycle and complete graphs $\lambda_{i}$ and $\mu_{i}$ can be obtained accurately and therefore analytical and simulation results are in complete agreement. In other structures we cannot obtain exact results, therefore we use a mean-field approach and coarse graining process. In these cases, simulation and analytical results are not exactly the same but show reasonable agreement.

\section{A. Cycle and complete graphs}

In a cycle graph it is easy to find $\lambda_{i}$ and $\mu_{i}$,

$$
\lambda_{i}=\frac{r}{N-i+r i}, \mu_{i}=\frac{1}{N-i+r i} .
$$

Figure 2 shows the fixation time versus network size in a cycle graph for three different fitness values. Solid lines and points show analytical and simulation results, respectively.

In a complete graph $\lambda_{i}$ and $\mu_{i}$ are obtained as

$$
\lambda_{i}=r \mu_{i}=\frac{r i(N-i)}{(N-1)(N-i+r i)} .
$$

Figure 3 shows the fixation time versus network size in a complete graph for three different fitness values. Complete

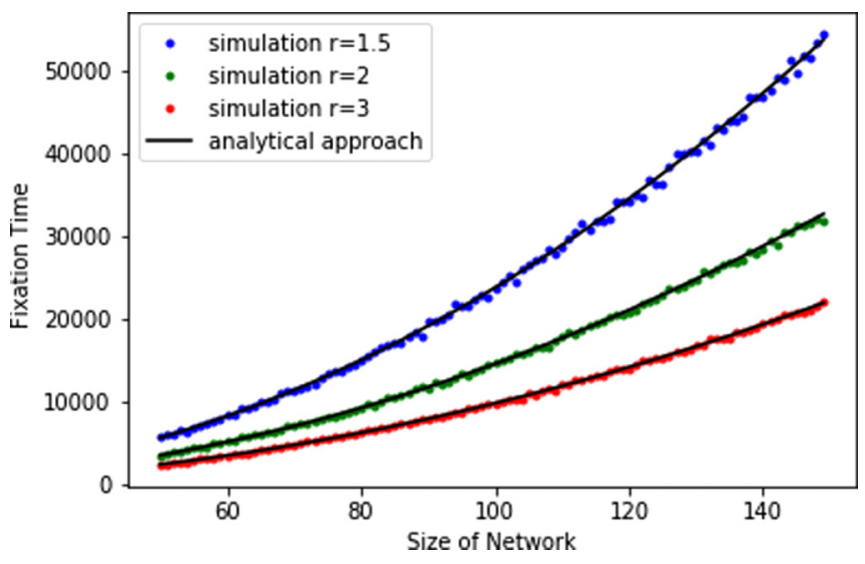

FIG. 2. Fixation time vs network size for a cycle graph with three different fitness values.

agreement of simulation and analytical results is obviously seen in these two figures. These results also confirm simulation results reported in Ref. [10].

\section{B. Erdös-Rényi network}

Now, we examine our method for a population of individuals which are connected to each other through a random (Erdös-Rényi) network [35] structure. Consider a random network with $N$ nodes. Each node is connected to every other node with probability $p$. The main step to find fixation time is to obtain $\lambda_{i}$ s and $\mu_{i}$ s. Unlike cycle and complete graphs, here we cannot obtain these parameters exactly, therefore, we use a mean-field approach to obtain them approximately.

Consider a random graph with $N$ nodes; an $i$ number of them are mutants. The probability for a mutant to be selected for reproduction is $\frac{r i}{N-i+r i}$. The average node degree of a randomly selected node in the Erdös-Rényi network is approximately $N p$. Now, a natural question is how many residents are a neighbor to a randomly selected mutant? At a first glance, one may suppose that there are $(N-i) p$ residents in the neighborhood of a randomly selected mutant, because there are $N-i$ residents in the network; each of them may be connected to the selected node with probability $p$. But,

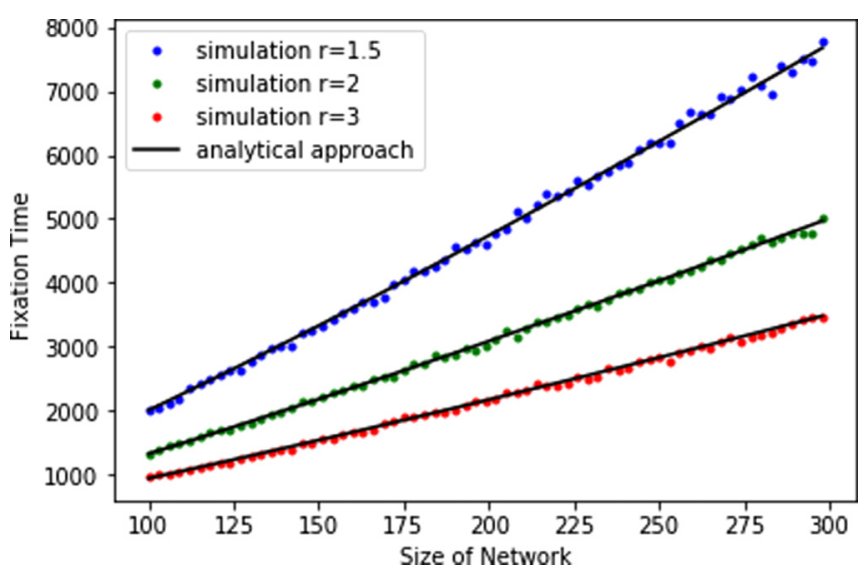

FIG. 3. Fixation time vs network size for a complete graph with three different fitness values. 


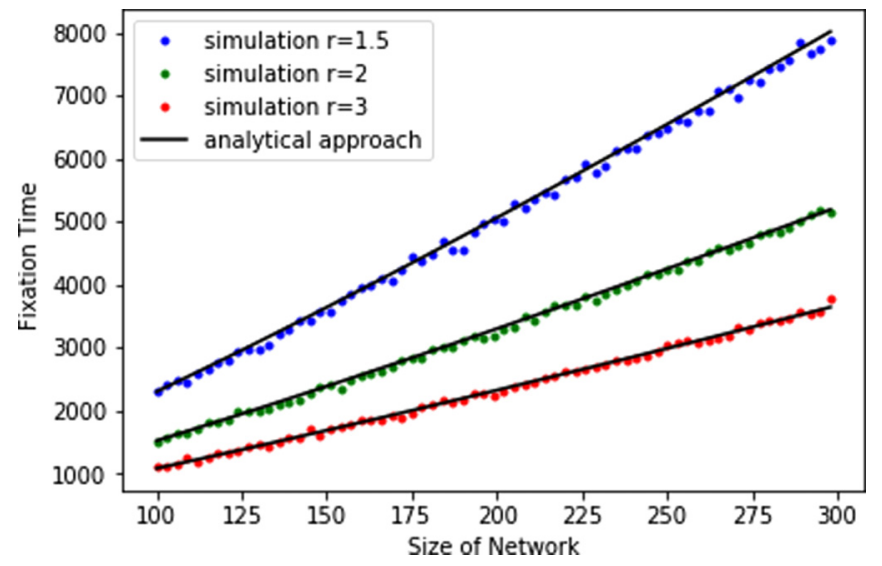

FIG. 4. Fixation time vs network size for an Erdös-Rényi network with three different fitness values. The connection probability is set to $p=0.16$.

one should note that the population of mutants grows more or less in a cluster form, therefore it is very likely for a chosen mutant to have at least two mutant neighbors. This argument becomes more accurate for smaller values of $p$. This means that the probability that a selected mutant is connected to a resident is $p-\frac{2}{N}$ and therefore the average number of residents connected to the selected mutant is $(N-i)\left(p-\frac{2}{N}\right)$. This determines the value of $\lambda_{i}$ as

$$
\lambda_{i}=\frac{r i}{N-i+r i} \frac{(N-i)\left(p-\frac{2}{N}\right)}{N p} .
$$

In the same way, one can obtain $\mu_{i}$,

$$
\mu_{i}=\frac{N-i}{N-i+r i} \frac{i\left(p-\frac{2}{N}\right)}{N p} .
$$

It is obvious from the above relations that in a random network, with this mean-field approximation, the condition $\lambda_{i}=r \mu_{i}$ is satisfied and therefore Eq. (8) can be used to calculate the fixation time. Figure 4 shows the fixation time versus network size for a random network with three different values of fitness. Figure 5 shows the fixation time versus $p$ in a random graph with size $N=100$. This figure shows that

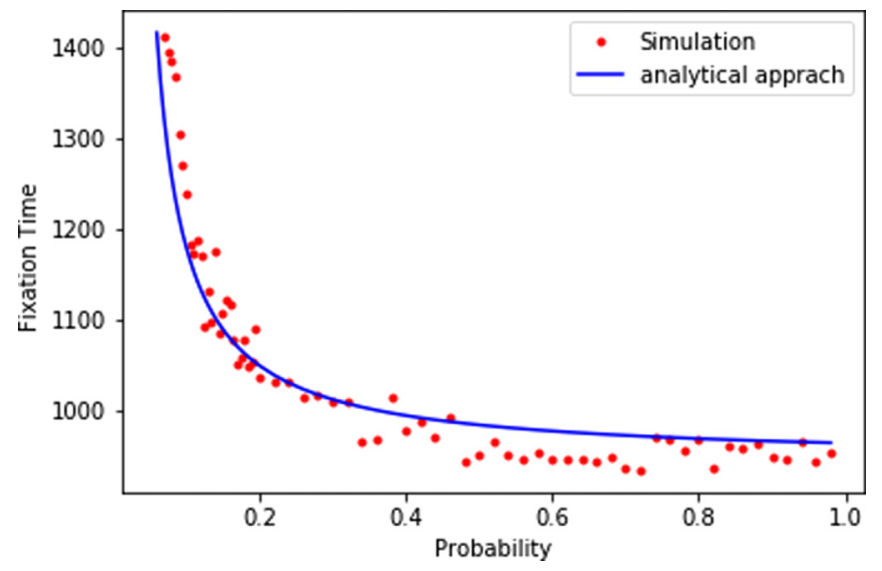

FIG. 5. Fixation time vs connection probability $p$ in an ErdösRényi graph with $N=100$ nodes and fitness $r=3$. the fixation time decreases with increasing the connection probability $p$ and approaches the fixation time of a complete graph for large values of $p$. This is in agreement with the results of Ref. [10] where exact results for a complete graph are reported.

\section{Regular graph}

Generally, a regular graph is a graph whose nodes are of the same degree. In this paper, we consider a regular graph with a circular structure with node degree $m$ (considered to be an even integer) which is constructed as follows: Put $N$ nodes on a circle and connect each node to its $\frac{m}{2}$ neighbors on each side.

Here again the probability for a mutant to be chosen for reproduction is $\frac{r i}{N-i+r i}$. Now, consider a configuration with $i$ mutants. Let $T_{i}$ denote the average number of edges connecting species of different types (interface edges). Each mutant has $m$ connections among them; $\frac{T_{i}}{i}$ are interface edges in average. Therefore the probability for a mutant offspring to substitute one of its resident neighbors is $\frac{\frac{T_{i}}{i}}{m}$ and consequently $\lambda_{i}$ reads

$$
\lambda_{i}=\frac{r i}{N-i+r i} \frac{\frac{T_{i}}{i}}{m}=\frac{r T_{i}}{m(N-i+r i)} .
$$

In the same manner $\mu_{i}$ is obtained as

$$
\mu_{i}=\frac{n-i}{N-i+r i} \frac{\frac{T_{i}}{n-i}}{m}=\frac{T_{i}}{m(N-i+r i)} .
$$

Enumeration of interface edges is used in other research for calculating $\lambda_{i}$ and $\mu_{i}$ in lattice networks [15]. By clustering growth assumption for mutants, the number of interface edges in both sides of a cluster is $T_{i}=\frac{m^{2}}{4}+\frac{m}{2}$. This is exact for $m=2$. For $m \geqslant 4$, it may happen that among the cluster of mutants, resident nodes appear as well. Direct calculation shows that the number of these residents is $\frac{m}{4}$ and since each one is connected to $m$ mutants, we should add $\frac{m^{2}}{4}$ to the number of interface edges. Therefore, for $m \geqslant 4$, we have $T_{i}=\frac{m^{2}}{2}+\frac{m}{2}$ and consequently

$$
\lambda_{i}=\frac{r\left(\frac{m}{2}+\frac{1}{2}\right)}{N-i+r i}, \quad \mu_{i}=\frac{\left(\frac{m}{2}+\frac{1}{2}\right)}{N-i+r i} .
$$

Substituting $\mu_{i}$ from Eq. (15) into Eq. (8) gives fixation time as a function of network size, fitness, and nodes degree. Figure 6 shows fixation time versus network size in a regular graph with $m=6$ and three fitness values.

\section{Scale-free network}

A scale-free network can be constructed by the BarabásiAlbert model [36]. In this model, one begins with a fully connected graph with $m_{0}$ nodes. Then, step by step new nodes are added to the network. A new node connects to $m<m_{0}$ existing nodes with a probability proportional to their degrees. This process continues until the desired network size is achieved. The degree distribution of this network is power law

$$
P(k)=\beta k^{-\gamma},
$$




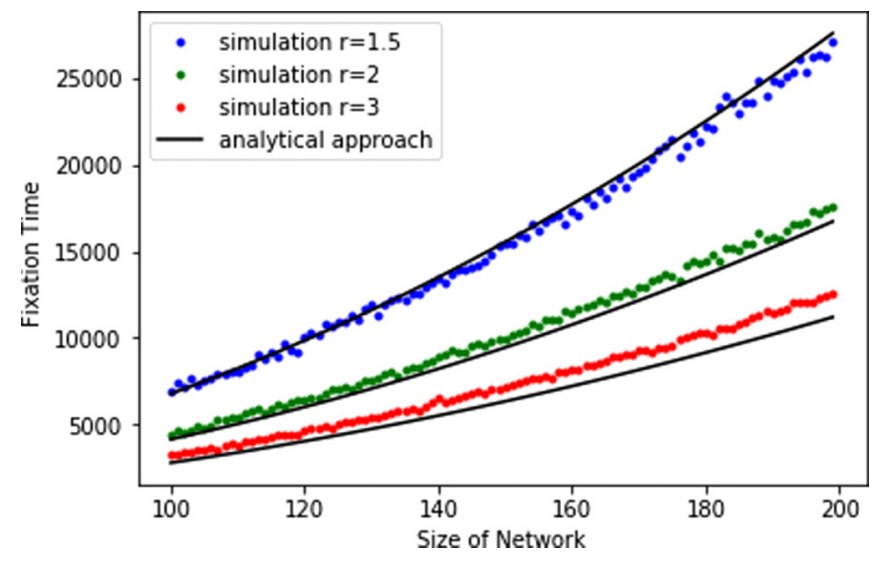

FIG. 6. Fixation time versus network size in a regular graph with $m=6$.

with $2<\gamma<3$. For large graphs $\gamma$ is approximately equal to 3. In Eq. (16) the minimum value of $k$ is $m$.

Dynamical processes like evolutionary dynamics or voter model on heterogeneous structures, e.g., scale-free networks, are vastly investigated. See for example $[37,38]$ just to mention a few.

Here, to approximate $\lambda_{i}$ and $\mu_{i}$ in a scale-free network, again we use the concept of interface edges. Let $T_{i}$ be the number of interface edges. Then, in analogy with the method used in regular networks, it is easily seen that

$$
\lambda_{i}=\frac{r T_{i}}{\langle k\rangle(N-i+r i)}, \quad \mu_{i}=\frac{T_{i}}{\langle k\rangle(N-i+r i)},
$$

where $\langle k\rangle$ is the average degree which is approximately equal to

$$
\langle k\rangle=\frac{\gamma-1}{\gamma-2} m
$$

for large network sizes.

Now, we have to find the average number of interface edges $T_{i}$. Suppose there are $i$ mutants and $T_{i}$ interface edges in the graph. Each resident node has at least $m$ edges and $\frac{T_{i}}{N-i}$ interface edges in average. Changing one resident to mutant adds 1 to the number of mutants. Consequently, it is easy to get the following recursion relation for the number of interface edges:

$$
T_{i+1}=T_{i}+m-\frac{T_{i}}{N-i}
$$

Assuming that $T_{0}=0$, this recursion relation is solved as

$$
T_{i}=m \sum_{j=1}^{i} \frac{N-i}{N-j}
$$

This, together with Eq. (17) gives us the approximate values of $\lambda_{i}$ and $\mu_{i}$. Inserting this $\mu_{i}$ into Eq. (8), we obtain the fixation time as a function of network size $N$, fitness $r$, and exponent $\gamma$. Figure 7 shows the average fixation time versus network size in a scale-free network.

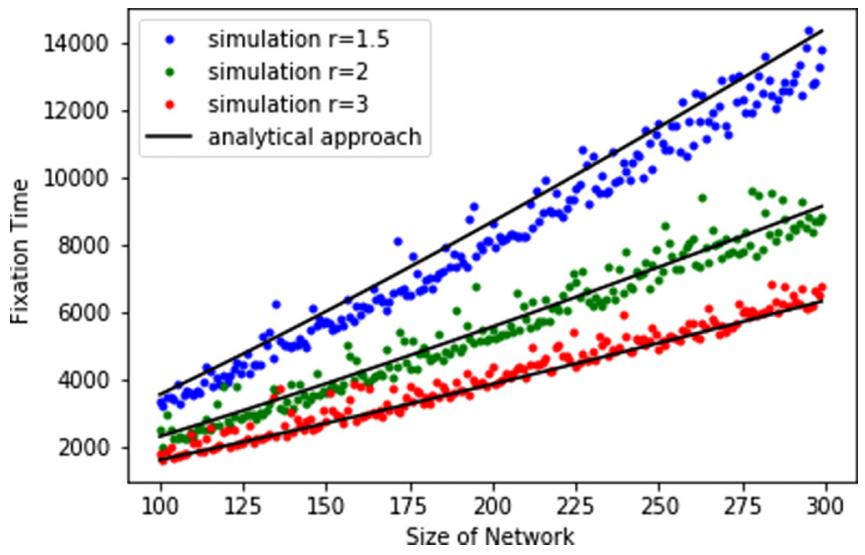

FIG. 7. Fixation time vs network size in a scale-free network with $m=6$ for three different fitness values.

\section{E. Small-world network}

Small-world networks are networks with small average shortest path length: starting from an arbitrary node on a small-world network, one can reach to other nodes through a small number of steps. The first model for generating a small-world graph introduced by Watts and Strogatz [39]. In this model, we begin with a regular graph and then, by rewiring probability $P_{\mathrm{ws}}$, rewire the edges. This procedure, with proper choices for $P_{\mathrm{ws}}$, leads to a graph with small-world property.

To find $\lambda_{i}$ and $\mu_{i}$ in a Watts-Strogatz graph, we again seek the average number of interface edges $\left(T_{i}\right)$,

$$
\lambda_{i}=\frac{r T_{i}}{m(N-i+r i)}, \quad \mu_{i}=\frac{T_{i}}{m(N-i+r i)} .
$$

In a regular graph, assuming clustering growth, we approximated the number of interface edges as $T_{i}=\frac{m^{2}}{2}+\frac{m}{2}$. For a Watts-Strogatz graph, we keep the clustering growth assumption, but not a single cluster. In fact, because of edge rewiring, it is very likely to have more than one cluster in the Moran process because every time the number of mutants increases by 1 , it is possible for the newborn mutant to appear in a new cluster through a rewired link. Therefore we suggest the following formula for the number of interface edges in a Watts-Strogatz graph:

$$
T_{i}=\left\langle c_{i}\right\rangle\left(\frac{m^{2}}{2}+\frac{m}{2}\right),
$$

where $\left\langle c_{i}\right\rangle$ is the average number of clusters in a graph with $i$ mutants, $\left\langle c_{i}\right\rangle=\sum P_{i}(c) c$. Here $P_{i}(c)$ is the probability for a graph with $i$ mutants to have $c$ clusters. By substituting (22) in Eq. (21), $\lambda_{i}$ and $\mu_{i}$ read

$$
\lambda_{i}=\frac{r\left\langle c_{i}\right\rangle\left(\frac{m}{2}+\frac{1}{2}\right)}{(N-i+r i)}, \mu_{i}=\frac{\left\langle c_{i}\right\rangle\left(\frac{m}{2}+\frac{1}{2}\right)}{(N-i+r i)} .
$$

To approximate $P_{i}(c)$, note that each node has $m$ edges in average. Therefore the maximum number of clusters is $R=\left\lfloor\frac{N}{m}\right\rfloor$ approximately, where $\lfloor$.$\rfloor is the floor function. Every$ time a new mutant is born, the probability for that to belong to a cluster different from that of its mother is $P_{\mathrm{ws}}$. With $i$ mutants this cluster changing happens $S:=\left\lfloor i P_{\mathrm{ws}}\right\rfloor+1$ times 


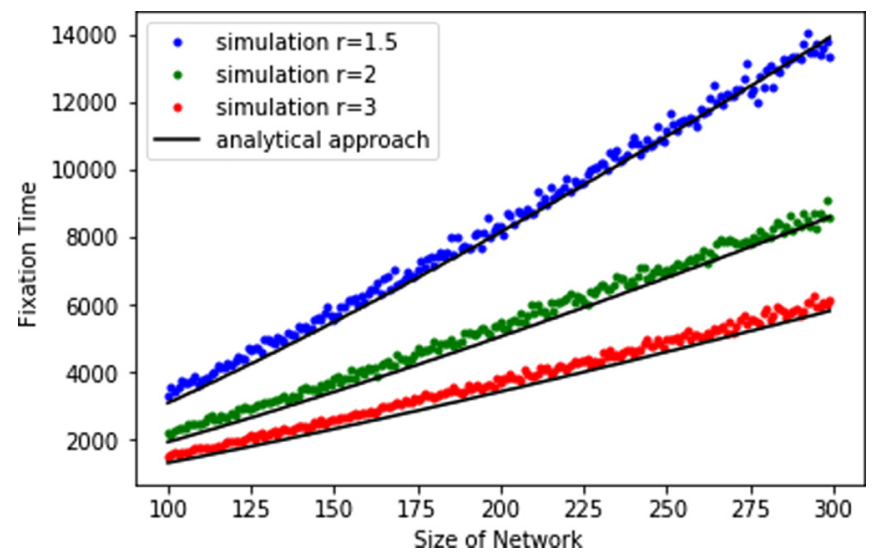

FIG. 8. Fixation time vs network size in Watts-Strogatz model with $\langle k\rangle=6$ and $P_{\mathrm{ws}}=0.1$.

approximately. Clearly, this is not equal to the number of clusters $c$. The question is how likely it is that $S$ times cluster changing leads to $c$ different clusters. Put another way, we want to select $S$ clusters among $R$ possible clusters (selection with replacement). What is the probability to get $c$ different clusters? Using simple combinatorics, one can see that this probability is

$$
P_{i}(c)=\frac{\alpha_{i}(c)}{\sum_{j=1}^{S} \alpha_{i}(j)},
$$

where

$$
\alpha_{i}(c)=\left(\begin{array}{l}
R \\
c
\end{array}\right)\left(\begin{array}{l}
S-1 \\
c-1
\end{array}\right) .
$$

One should note that the approximation scheme used above works well when the number of mutants $i$ is small, because the number of clusters itself is not an exact variable, as well as the number of cluster changing. Therefore, the smaller number of cluster changing, the better the approximation. On the other hand, it is clear that the number of average interface edges between $i$ mutants and $N-i$ residents is equal to the number of interface edges between $N-i$ mutants and $i$ residents, i.e., $T_{i}=T_{N-i}$. Therefore, we will use $T_{N-i}$ instead of $T_{i}$ whenever $i>\frac{N}{2}$. Figure 8 shows analytical and simulation results for a Watts-Strogatz graph with $m=6$ and $P_{\text {ws }}=0.1$. Here again we see a good agreement between simulation and analytical approximation.

\section{SUMMARY AND CONCLUDING REMARKS}

To summarize, we used an analytical method to calculate fixation and extinction times of a birth-death Moran process on a general evolutionary graph. This method is based on Markov chains and in principle is applicable to all kinds of complex structures. Results of this method are in agreement with results obtained by recursive equation methods and are confirmed with simulation results for the Moran process on many structured populations. This method can easily be used for a dynamical process with more than two absorption states (for example a population with more than two types of species) and provides a straightforward tool to calculate all absorption times.

\section{ACKNOWLEDGMENTS}

The authors would like to thank M. Askari for fruitful discussion and comments. They also appreciate valuable comments from anonymous referees which substantially helped improve the paper.
[1] M. A. Nowak, Evolutionary Dynamics (Harvard University Press, Cambridge, MA, 2006).

[2] E. Lieberman, C. Hauert, and M. A. Nowak, Nature (London) 433, 312 (2005).

[3] P. A. P. Moran, Math. Proc. Cambridge Philos. Soc. 54, 60 (1958).

[4] P. Shakarian, P. Roos, and A. Johnson, Biosystems 107, 66 (2012).

[5] M. Broom and J. Rychtar, Proc. R. Soc. London, Ser. A 464, 2609 (2008).

[6] M. Frean, P. B. Rainey, and A. Traulsen, Proc. R. Soc. London, Ser. B 280, 20130211 (2013).

[7] L. Hindersin, M. Moeller, A. Traulsen, and B. Bauer, Biosystems 150, 87 (2016).

[8] L. Hindersin and A. Traulsen, J. R. Soc. Interface 11, 20140606 (2014).

[9] M. Askari and K. A. Samani, Phys. Rev. E 92, 042707 (2015).

[10] M. Askari, Z. Moradi Miraghaei, and K. Aghababaei Samani, J. Stat. Mech.: Theory Exp. (2017) 073501.

[11] S. Giaimo, J. Arranz, and A. Traulsen, PLoS Comput. Biol. 14, e1006559 (2018).

[12] F. Alcalde Cuesta, P. Gonzalez Sequeiros, and A. Lozano Rojo, PLoS One 13, e0200670 (2018).
[13] A. Pavlogiannis, J. Tkadlec, K. Chatterjee, and M. A. Nowak, Sci. Rep. 7, 82 (2017).

[14] M. Broom, J. Rychtar, and B. T. Stadler, J. Stat. Theory Pract. 5, 369 (2011).

[15] C. J. Paley, S. N. Taraskin, and S. R. Elliott, Phys. Rev. Lett. 98, 098103 (2007).

[16] O. Hauser, A. Traulsen, and M. A. Nowak, J. Theor. Biol. 343, 178 (2014).

[17] B. Allen, M. A. Nowak, and U. Dieckmann, Am. Nat. 181, E139 (2013).

[18] P. Ashcroft, C. Smith, M. Garrod, and T. Galla, J. Theor. Biol 420, 232 (2017).

[19] G. Szabo and G. Fath, Phys. Rep. 446, 97 (2007).

[20] C. P. Roca, J. A. Cuesta, and A. Sanchez, Phys. Life Rev. 6, 208 (2009).

[21] A. J. Bladon, T. Galla, and A. J. McKane, Phys. Rev. E 81, 066122 (2010).

[22] M. Assaf and B. Meerson, J. Phys. A 50, 263001 (2017).

[23] L. M. Ying, J. Zhou, M. Tang, S. G. Guan, and Y. Zou, Front. Phys. 13, 130201 (2018).

[24] T. Monk, P. Green, and M. Paulin, Proc. R. Soc. London, Ser. A 470, 20130730 (2014). 
[25] P. M. Altrock, A. Traulsen, and M. A. Nowak, Phys. Rev. E 95, 022407 (2017).

[26] L. Hindersin and A. Traulsen, PLOS Comput. Biol. 11, e1004437 (2015).

[27] J. P. Gleeson, J. A. Ward, K. P. O'Sullivan, and W. T. Lee, Phys. Rev. Lett. 112, 048701 (2014).

[28] L. Weng, A. Flammini, A. Vespignani, and F. Menczer, Sci. Rep. 2, 335 (2012).

[29] K. I. Goh, D. S. Lee, B. Kahng, and D. Kim, Phys. Rev. Lett. 91, 148701 (2003).

[30] P.-A. Noël, C. D. Brummitt, and R. M. D’Souza, Phys. Rev. Lett. 111, 078701 (2013).

[31] C. Castellano, S. Fortunato, and V. Loreto, Rev. Mod. Phys. 81, 591 (2009).
[32] C. M. Grinstead and J. L. Snell, Introduction to Probability (American Mathematical Society, Providence, RI, 1997).

[33] C. M. da Fonseca and J. Petronilho, Linear Algebra Appl. 325, 7 (2001).

[34] T. Antal and I. Scheuring, Bull. Math. Biol. 68, 1923 (2006).

[35] P. Erdös and A. Rényi, Publ. Math. Debrecen 6, 290 (1959).

[36] A. L. Barabasi, R. Albert, and H. Jeong, Physica A (Amsterdam) 272, 173 (1999).

[37] T. Antal, S. Redner, and V. Sood, Phys. Rev. Lett. 96, 188104 (2006).

[38] V. Sood, T. Antal, and S. Redner, Phys. Rev. E 77, 041121 (2008).

[39] D. J. Watts and S. H. Strogatz, Nature (London) 393, 440 (1998). 